\title{
A NOTE ON BELTRAMI AND COMPLEX-LAMELLAR FLOWS BEHIND A THREE-DIMENSIONAL CURVED GASDYNAMIC SHOCK WAVE*
}

\author{
By D. J. TEMBHAREY (D. B. Science College, Gondia (M. S.), India) \\ AND \\ S. K. SACHDEVA (Government College, Balaghat (M. P), India)
}

Introduction. In this communication we derive the conditions for the existence of complex-lamellar and Beltrami flows behind a three-dimensional stationary curved shock wave. It is assumed that the flow ahead of the shock is uniform and known and that dissipative mechanisms such as viscosity and thermal conduction are absent.

Let $u_{i}, \rho$ and $p$ denote respectively the components of velocity, density and pressure of the gas. The unit vector $X_{i}$ normal to the shock is assumed continuously differentiable and directed downstream. Furthermore, we write

$$
\begin{gathered}
u_{i, j} x_{i \alpha}=A_{i \alpha}, \quad p_{. i} x_{i \alpha}=B_{\alpha}, \\
F_{\alpha}=F_{i} x_{i \alpha}, \quad F_{\eta}=F_{i} X_{i},
\end{gathered}
$$

where $x_{i \alpha} \equiv \partial x_{i} / \partial y^{\alpha}$; the $x_{i}(i=1,2,3)$ and $y^{\alpha}(\alpha=\mathrm{I}$, II $)$ denote respectively the Cartesian coordinates and the Gaussian coordinates of a point.

The expression for the vorticity $W_{i}$ generated behind the shock can be derived from the momentum equation by the method given in [1] as

$$
W_{i}=\left(-\epsilon^{\alpha \lambda} x_{i \lambda} / \rho u_{n}\right)\left(B_{\alpha}+\rho u^{\beta} A_{i \beta} x_{j \alpha}+\rho u_{n} A_{i \alpha} X_{j}\right)
$$

where $\epsilon^{\alpha \lambda}$ are the components of the surface permutation tensor.

If we define the shock strength $\Delta$ by $[\rho]=\Delta \rho_{1}$, where the notation $[F] \equiv F-F_{1}, F_{1}$ and $F$ being the values of $F$ just in front of and behind the shock respectively, the jump conditions read

$$
\begin{gathered}
{\left[u_{i}\right]=-\Delta u_{1 n} X_{i} /(1+\Delta),} \\
{[p]=\Delta \rho_{1} u_{1 n} /(1+\Delta) .}
\end{gathered}
$$

2. Complex-lamellar and Beltrami flows behind a stationary shock wave. The flow is called complex-lamellar if $u_{i} W_{i}=O$ [2]. Differentiating (3) and (4) with respect to $y^{\alpha}$ and substituting the values of $A_{i \alpha}$ and $B_{\alpha}$ thus obtained in (2), we obtain

$$
W_{i}=-\left(\Delta^{2} /(1+\Delta)\right) \epsilon^{\alpha \lambda} x_{i \lambda} u_{i \gamma} a^{\beta \gamma} b_{\alpha \beta}
$$

* Received June 10, 1972; revised version received September 16, 1972. The authors wish to thank the reviewer for his helpful criticism. The research of the first-named author was partially supported by the University Grants Commission, India. 
where $a_{\alpha \beta}$ and $b_{\alpha \beta}$ denote respectively the first and second fundamental forms of the shock surface. Furthermore, we choose the lines of curvature as the Gaussian coordinate curves on the shock surface and consequently obtain (5) in the form

$$
W_{i}=\left(\Delta^{2} / \sqrt{ } a(1+\Delta)\right)\left(x_{i \mathrm{I}} k_{\mathrm{II}} u_{\mathrm{II}}-x_{i \mathrm{II}} k_{\mathrm{I}} u_{\mathrm{I}}\right)
$$

where $a=\operatorname{det}\left\|a_{\alpha \beta}\right\|$. In deriving (6) we have used the formulas $X_{i, \alpha}=-a^{\gamma \beta} b_{\alpha \gamma} x_{i \beta}=$ $-k_{\alpha} x_{i \alpha}, \alpha$ unsummed, where $k_{\mathrm{I}}$ and $k_{\mathrm{II}}$ are the principal normal curvatures of the shock surface.

Multiplying (6) by $u_{i}$ and summing, we obtain

$$
u_{\imath} W_{i}=\left(\Delta^{2} / \sqrt{ } a(1+\Delta)\right) u_{\mathrm{I}} u_{\mathrm{II}}\left(k_{\mathrm{II}}-k_{\mathrm{I}}\right) .
$$

Thus the flow behind the shock will be complex-lamellar if at least one of the following conditions holds:

$$
\text { (C1) } K_{\mathrm{I}}=K_{\mathrm{II}}, \quad \text { (C2) } u_{\mathrm{I}}=0 ; \quad \text { (C3) } u_{\mathrm{II}}=0 .
$$

Condition (C1) implies that the flow behind an oblique spherical shock is complexlamellar, and conditions (C2) and (C3) together imply that the flow behind a normal shock is complex-lamellar. For a plane shock, $K_{\mathrm{I}}=K_{\mathrm{II}}=0$, and consequently we obtain the result that the flow behind a plane shock is complex-lamellar.

The velocity field is said to be a Beltrami field if $\epsilon_{i j k} u_{i} W_{i}=0$ [2]. Multiplying (6) by $\epsilon_{i j k} u_{i}$ and summing, we obtain

$$
\begin{aligned}
\lambda_{k} \equiv & \epsilon_{i j k} u_{i} W_{i} \\
= & \left(\Delta^{2} / \sqrt{ } a(1+\Delta)\right)\left\{\left(u_{\mathrm{II}} k_{\mathrm{II}} x_{1 \mathrm{I}}-u_{\mathrm{I}} k_{\mathrm{I}} x_{1 \mathrm{II}}\right)\left(\epsilon_{21 k} u_{2}+\epsilon_{31 k} u_{3}\right)\right. \\
& +\left(u_{\mathrm{II}} k_{\mathrm{II}} x_{2 \mathrm{I}}-u_{\mathrm{I}} k_{\mathrm{I}} x_{2 \mathrm{II}}\right)\left(\epsilon_{12 k} u_{1}+\epsilon_{32 k} u_{3}\right) \\
& \left.+\left(u_{\mathrm{II}} k_{\mathrm{II}} x_{3 \mathrm{I}}-u_{\mathrm{I}} k_{\mathrm{I}} x_{3 \mathrm{II}}\right)\left(\epsilon_{13 k} u_{1}+\epsilon_{23 k} u_{2}\right)\right\} .
\end{aligned}
$$

Thus in order that $\lambda_{k}=0$, at least one of the following conditions must be satisfied:

(B1) $k_{\mathrm{I}}=k_{\mathrm{II}}=0$; i.e. the shock surface is a plane,

(B2) $u_{\mathrm{I}}=u_{\mathrm{II}}=0$; i.e. the flow is normal to the shock,

(B3) $k_{\mathrm{I}}=u_{\mathrm{II}}=0$

(B4) $k_{\mathrm{II}}=u_{\mathrm{I}}=0$.

Condition (B2) implies that the flow behind a normal shock is a Beltrami flow. Conditions (B3) and (B4) lead to the same result. In (B4), $k_{\mathrm{II}}=0$ implies that the shock surface is a developable surface and as such its generators and their orthogonal trajectories form its two congruences of lines of curvatures [3a], while $u_{\mathrm{I}}=0$ implies that the orthogonal trajectories to its generators are plane curves lying in the planes which are normal to the direction of the given uniform flow in front of the shock wave.

Now, with the exception of cylinders and cones, every developable surface is the tangent surface of some curve [3b] and the orthogonal trajectories of the tangent surface of a curve are the involute of the curve [3c]. But the necessary and sufficient condition that the involutes of a twisted curve be plane curves is that the curve be a cylindrical helix [3d]. Moreover, the planes of the involutes of a cylindrical helix are normal to the generators of the cylinder on which the helix lies [3e]. Therefore, if we take the helix to lie on the cylinder whose generators are in the direction of the flow, then the tangent surface of this helix, developable helicoid, satisfies condition (B4). As far 
as a cone and a cylinder are concerned it can be easily seen that the right circular cone with its axis parallel to the direction of the uniform flow and any cylinder with generators parallel to the direction of the uniform flow satisfy condition (B4). We thus get the result: the flow behind an oblique shock is a Beltrami flow if the shock surface is a plane, a right circular cone, a cylinder or a developable helicoid. Furthermore, as observed by Kanwal [4], they are the only surfaces behind which the flow remains irrotational.

\section{REFERENCES}

[1] R. P. Kanwal, Determination of vorticity and gradients of flow parameters behind a three-dimensional unsteady curved shock wave, Arch. Ratl. Mech. Anal. 1, 225-232 (1958)

[2] R. Aris, Vectors, tensors and the basic equations of fluid mechanics, Prentice-Hall, Inc., 1962, p. 64

[3] L. P. Eisenhart, Introduction to differential geometry, Princeton University Press, 1941, (a) chapter 4, (b) p. 54 (c) p. 39 , (d) p. 38 , (e) p. 37

[4] R. P. Kanwal, On curved shock waves in three-dimensional gas flows, Quart. Appl. Math. 16, 361-372 (1959) 